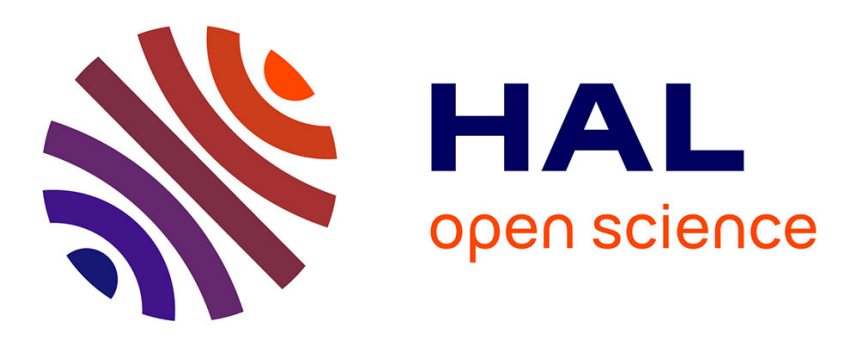

\title{
Authentication Using Metallic Inkjet-Printed Chipless RFID Tags
}

Zeshan Ali, Etienne Perret, Nicolas Barbot, Romain Siragusa, David Hely, Maxime Bernier, Frédéric Garet

\section{- To cite this version:}

Zeshan Ali, Etienne Perret, Nicolas Barbot, Romain Siragusa, David Hely, et al.. Authentication Using Metallic Inkjet-Printed Chipless RFID Tags. IEEE Transactions on Antennas and Propagation, 2020, 68 (5), pp.4137 - 4142. 10.1109/TAP.2019.2948740 . hal-02337466

\section{HAL Id: hal-02337466 https://hal.science/hal-02337466}

Submitted on 13 Jan 2020

HAL is a multi-disciplinary open access archive for the deposit and dissemination of scientific research documents, whether they are published or not. The documents may come from teaching and research institutions in France or abroad, or from public or private research centers.
L'archive ouverte pluridisciplinaire HAL, est destinée au dépôt et à la diffusion de documents scientifiques de niveau recherche, publiés ou non, émanant des établissements d'enseignement et de recherche français ou étrangers, des laboratoires publics ou privés. 


\title{
Authentication Using Metallic Inkjet-Printed Chipless RFID Tags
}

\author{
Zeshan Ali, Etienne Perret, Senior Member, IEEE, Nicolas Barbot, \\ Romain Siragusa, David Hély, Maxime Bernier, and Frédéric Garet
}

\begin{abstract}
A high-level (i.e., in terms of security) authentication method is proposed in this paper, where the chipless radio frequency identification (RFID) is extended to the chipless authentication. The proposed method is based on low cost inkjet-printed square check patterned chipless tags, whose design is explicitly optimized keeping the randomness inherent to the inkjet printing process in view. These optimized chipless tags are very difficult to duplicate, as their unique backscattered electromagnetic (EM) responses depend on the proximate coupling among the possible separated squares, which is happened naturally due to the randomness in inkjet printing. The performance of the proposed method is analyzed by a low cost impulse radio (IR) ultrawideband (UWB) chipless RFID reader as well as by the highly accurate vector network analyzer (VNA) based chipless RFID reader. The achieved probability of error is comparable to the various fingerprint evaluation campaigns found in the literature.
\end{abstract}

Index Terms-Authentication technology, fabrication tolerance, fully printable chipless RFID, inkjet printing, radar approach, randomness.

\section{INTRODUCTION}

Counterfeiting is a significant threat to manufacturers and also a concern for human health [1]. Numerous countermeasures have been developed in recent times to counter this menace: overt features (low security level), covert features (medium security level) and forensic methods (high security level) [1]. To develop highly secure authentication techniques, natural randomness can be exploited. Some examples of natural randomness based authentication techniques are the random bubble patterns [2], and the random wires patterns [3]. In the literature, efforts have been made to present the product authentication capabilities by using chipless radio frequency (RF) identification (RFID). These authentication methods are developed by using, for example, dipoles [4], a 2D print comprising optical code as well as electromagnetic (EM) fingerprint [5], concentric ring slot resonators [6] (only simulations results), the split ring resonators [7], and RF absorbing markers [8]. In our previous work, we have demonstrated the possibility of chipless RFID based authentication. This potential has been demonstrated by purposely applying the dimensional variations along geometrical dimensions of C-folded scatterers of printed circuit board (PCB) tags [9]-[11]. Subsequently, the concept of chipless authentication using solely natural process variations inherent to PCB realization technology has been proved in [12]. Two populations of chipless tags (each comprising forty-five tags) are realized intermittently with individual film masks (i.e., using same technology from the same company).

Manuscript received May 22, 2019; revised September 19, 2019; accepted October 6, 2019. This work was supported in part by the Univ. Grenoble Alpes, in part by the Région Auvergne-Rhône-Alpes, and in part by the Institut Universitaire de France. This project has also received funding from the European Research Council (ERC) under the European Union's Horizon 2020 Research and Innovation Program (grant agreement No 772539).

Z. Ali, N. Barbot, R. Siragusa, and D. Hély are with the Univ. Grenoble Alpes, Grenoble INP, LCIS, 26000 Valence, France (e-mail: zeshan.ali@lcis.grenoble-inp.fr).

E. Perret is with the Univ. Grenoble Alpes, Grenoble INP, LCIS, 26000 Valence, France, and also with the Institut Universitaire de France, 75005 Paris, France.

M. Bernier and F. Garet are with the Université Savoie Mont Blanc, CNRS, Grenoble INP, IMEP-LAHC, 73376 Le Bourget-du-Lac, France.
The contributions of natural randomness from two film masks and PCB realization process have been presented by in-depth statistical analysis of the comparisons of free space measurements and by microscopic measurements of physical dimensions of chipless tags of both populations. In this PCB chipless tags based authentication method, the authenticity information resides around the peak apex which carries primarily the aspect-independent parameters: frequency of resonance $f_{r}$ and quality factor $Q$. By virtue of natural randomness, in PCB chipless tags, the maximum variation of peak apex of backscattered signals is $94 \mathrm{MHz}$.

This paper is introducing a high-level (i.e., in terms of security) authentication method, where chipless authentication using natural randomness inherent to the inkjet printing process is presented. The chipless RFID tags printed using a low cost off-the-shelf inkjet printer with metallic ink are utilized. The design of chipless tags is explicitly optimized for inkjet printing process based on the resolution of the inkjet printer. For the first time, randomness inherent to the inkjet printer fabrication is used for authentication based on backscattering signature of a simple label. The proposed chipless authentication based on inkjet-printed chipless tags is a practical solution than the chipless authentication based on PCB chipless tags discussed in [12]. PCB realization technology is chosen in [12] to make a point of reference for the performance given by simple realizations using well-known PCB fabrication method. As compared to PCB chipless tags, the proposed inkjet-printed chipless tags are optimized such that the peak apexes of backscattered signals can change up to $4.48 \mathrm{GHz}$ in the range of ultrawideband (UWB). Therefore, the randomness due to the structure sensitivity regarding the fabrication process is considerably increased in this work.

The proposed authentication method is possibly low cost, directly printable or easy to fabricate, possibly non-invasive or nondestructive, difficult to duplicate, and simple in operation. Furthermore, the chipless tags can entirely be hidden (potentially without performance degradation) for the confidentiality and the product integrity of high-end luxury products, as compared to the top layer (3D structures) [13] or the fibers (2D) [14] which can deteriorate the product integrity by their external aspects. In this paper, multiple populations of chipless RFID tags are realized, where each population is constituted of thirty inkjet-printed chipless tags exhibiting natural process randomness. The performance of the proposed authentication method is analyzed by a low cost impulse radio (IR) UWB chipless RFID reader as well as by a highly accurate vector network analyzer (VNA) based chipless RFID reader. This paper is organized as follows. Section II explains the natural randomness inherent to the inkjet printing process. Section III is dedicated to the optimization of design of conventional C-folded scatterer to elevate its dimensional sensitivity. Section IV discusses the further optimization of design for inkjet printing, and realization of chipless RFID tags. Sections V provides the validation of the proposal by presenting the performance of optimized chipless tag (with natural randomness) using a low cost IR-UWB chipless RFID reader and using a highly accurate VNA based chipless RFID reader. Section VI presents the flow of implementation of proposed chipless authentication. Finally, Section VII presents the conclusions. 


\section{NATURAL RANDOMNESS INHERENT TO INKJET PRINTING}

For inkjet printing technology, the print quality is vitally dependent on the chosen resolution. Apart from this, numerous factors are also involved that determine the print quality such as the design size and shape, the ink density and type, the substrate texture, the drop position accuracy, and the type of image [15]. For instance, a straight line can also have random variations along its edge [16]. As another example, the thickness of an inkjet-printed layer is in the order of a few $\mu \mathrm{m}$ [17], as compared to $17.5 \mu \mathrm{m}$ or $35 \mu \mathrm{m}$ for conventional PCB microstrip trace. Furthermore, in [18], a layout has been presented to explain a change in the width of output inkjetprinted layer from the width of input digital design.

To exploit process randomness inherent to the inkjet-printing technology for chipless authentication, we adopted an optimization of the design of chipless tags to elevate their sensitivity to the randomness inherent to the inkjet printing. Fig. 1 is a zoom photograph of one of our realization. It can be seen that from an input digital mask comprising vertex-to-vertex separated square geometries (i.e., supplied to the inkjet printer), a randomly connected or disconnected realization can be printed. It is important to note that these random variations are happening during the fabrication process (inkjet printing) but not from the input digital mask. Therefore, at low operating frequency, simple vertex-to-vertex adjacent metallic geometries can behave like electrical switches as depicted in Fig. 1. In UWB, these random vertex-to-vertex separations among metallic geometries provide a proximate coupling effect to generate unique EM signals. Such a vertex-to-vertex adjacent design can be realized from a simple square check pattern to prove the concept. Next, the square check patterns will be incorporated to optimize the design of chipless RFID tags to be employed for authentication.

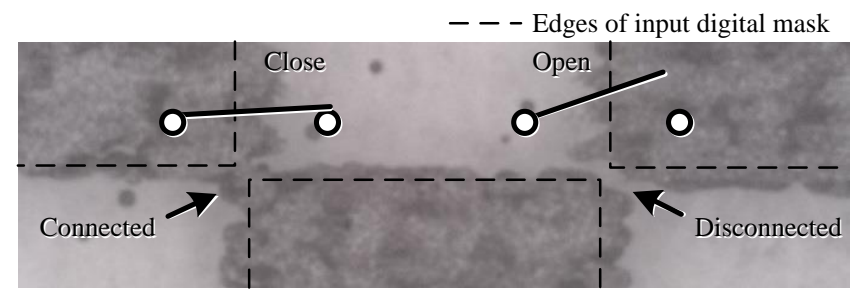

Fig. 1. Example of realization to show an analogy of vertex-to-vertex adjacent metallic geometries to open and close states of electrical switches.

\section{OPTIMIZATION OF CHIPLESS RFID TAGS}

A resonant structure for the design of chipless RFID tag might be a better choice than a non-resonant structure because of several reasons: (i) its response is dependent on its geometrical dimensions and polarization of plane wave. (ii) it provides better signal to noise ratio (SNR). (iii) it produces a decaying time-domain (TD) response which is beneficial for extracting the tag's response. A C-folded scatterer is a coplanar stripline (CPS) [19]. Transmission line analysis of such a C-folded scatterer has been discussed in [20]. For chipless authentication, the potential of conventional $\mathrm{C}$-folded scatterer [see Fig. 2(a)] based chipless tags using PCB technology has been discussed in [9]-[12]. The frequency of resonance $f_{r}$ of such CPS C-folded scatterer based chipless tags is expressed as [20]:

$$
f_{r}=\frac{c}{4 L^{\prime} \sqrt{\varepsilon_{e f f}}}
$$

where $L^{\prime}$ corresponds to the total length of each arm (i.e., a summation of physical length $L$ and complementary length $\Delta L$ which is added to compensate the fringing fields) and $\varepsilon_{e f f}$ corresponds to the effective permittivity of the substrate. For the rest of this paper, $L^{\prime}$ and $L$ are used interchangeably for the simplicity in the discussion. With fixed values of the trace width $w$, the metal thickness $t$, the substrate thickness $h$, and the permittivity of the substrate $\varepsilon_{r}$, two dimension parameters of a C-folded scatterer take part in the calculation of its EM response [see Fig. 2(a)]: the length $L$ (or alternatively $L^{\prime}$ ) as it is directly related to $f_{r}$ in (1) and the spacing between two arms $g$ as it is related to $\varepsilon_{\text {eff }}$ (for details about the calculation of $\varepsilon_{e f f}$, see [19]). Such a dependence of response on the geometrical dimensions is very suitable for the employment of square check pattern to optimize the design of classical C-folded scatterer for authentication application. Fig. 2 provides the concept of optimization from the design of conventional C-folded scatterer [Fig. 2(a)] to the sensitive C-folded scatterer [Fig. 2(b)]. For a Cfolded scatterer, the radar cross section (RCS) is directly associated with the spacing between the two arms $g$ as discussed in [21] with a C-folded scatterer presenting comparable dimension as shown in Fig. 2. For this reason, the spacing between the two arms $g$ is increased to a value of $3.5 \mathrm{~mm}$ in the design of the optimized tags compared to the conventional design discussed in [10]. For the demonstration of optimization, the length $L$ of the conventional Cfolded scatterer is divided into two parts: the primary length $L_{1}$ and the secondary length $L_{2}$. Fig. 2(b) shows the layout of the optimized design of C-folded scatterer exhibiting five squares check patterns along the secondary length of arms $L_{2}$. The basis of choosing the lengths $L_{1}=5.35 \mathrm{~mm}$ and $L_{2}=17.15 \mathrm{~mm}$ is to keep the frequency band of operation of $\mathrm{C}$-folded scatterer within UWB which is compatible with IR-UWB chipless RFID reader [22].

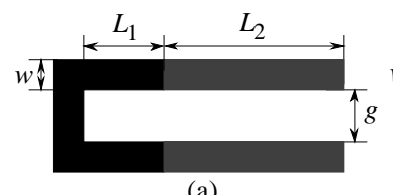

(a)

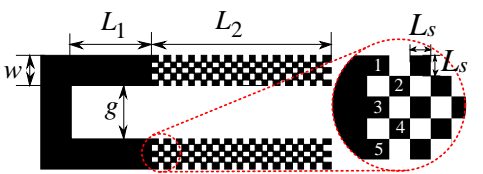

(b)
Fig. 2. Layouts of the C-folded scatterers. (a) Conventional design of the Cfolded scatterer. (b) Optimized design of the $\mathrm{C}$-folded scatterer exhibiting five squares check pattern. $L_{1}=5.35 \mathrm{~mm}, L_{2}=17.15 \mathrm{~mm}, w=2 \mathrm{~mm}, g=3.5 \mathrm{~mm}$, and length (and width) of each square geometry $L_{s}=0.4 \mathrm{~mm}$.

The substrate used in this work is the Polyethylene terephthalate (PET) printable film (Novele ${ }^{\mathrm{TM}}$ IJ-220) with a thickness $t=140 \mu \mathrm{m}$. For each chipless tag, the substrate's dimensions are $48 \times 48 \mathrm{~mm}^{2}$. The electrical characterization this PET printable film is conducted using the cavity resonator from Damaskos, INC, yields the relative permittivity $\varepsilon_{r}=2.99$ and dielectric loss tangent $\tan \delta=0.015$. Such a PET based substrate is chosen because of its low cost, low loss, and directly printable properties.

For simulations of the layouts of C-folded scatterers presented in Fig. 2 are illuminated by a plane wave using a full-wave EM simulator (CST MWS). The simulations results show that the values of $f_{r}$ for the optimized design of C-folded scatterer [Fig. 2(b)] are $3.4 \mathrm{GHz}$ and $6.5 \mathrm{GHz}$ while $L_{1}$ and $L_{2}$ are connected and disconnected, respectively. To disconnect $L_{1}$ and $L_{2}$, we separated them by a spacing of $50 \mu \mathrm{m}$ (where $50 \mu \mathrm{m}$ is compensated from $L_{2}$ ). This frequency band of variation (i.e., $3.4 \mathrm{GHz}-6.5 \mathrm{GHz}$ ) of the optimized design of C-folded scatterer is compatible with UWB.

To demonstrate the sensitivity of optimized scatterer, we purposely shortened the length of first and fifth square elements of lower arm [see the inset of Fig. 2(b)] by $50 \mu \mathrm{m}$ to introduce disconnections, successively. Simulations results show that the applied reduction on the length of first and fifth square elements produces a frequency shift $\Delta f_{r}^{1 \text { st }}=11.9 \mathrm{MHz}$ and $\Delta f_{r}^{5 \text { th }}=6.3 \mathrm{MHz}$, respectively. It can be observed that $\Delta f_{r}^{1 \text { st }}$ is larger than $\Delta f_{r}^{5 \text { th }}$. This 
is because the $\mathrm{C}$-folded scatterer behaves like a slot with a high concentration of the current density in the interior region than the exterior region of the two arms (see [23, Figs. 3 and 4]). In practice, the optimized scatterer [Fig. 2(b)] would be sensitive to each existing square geometry of the check pattern. The natural variations would be due to the possibility to have random electrical conductivity among adjacent square geometries depending on the random positions of ink drops in the inkjet printing as depicted in Fig. 1. It would ultimately produce unique patterns to be appropriate for authentication. These unique tags are very difficult to duplicate, as the backscattered EM responses depend on the proximate coupling among the possible separated squares, which is happened due to randomness in the inkjet printing.

\section{Further Optimization of Design AND InKJET PRINTING OF ChIPLESS RFID TAGS}

For the realization of square check patterned C-folded scatterer chipless tags, we used Epson C88+ printer with JS-B25P silver conductive ink without annealing process. We have testified that the inkjet printing of the input digital mask of scatterer with vertex-tovertex adjacent check pattern [see Fig. 2(b)] always produces an output with well-connected check pattern. This behavior is reducing the opportunity of exploiting the randomness of inkjet printing for authentication purposes. For this reason, further optimization of the input digital mask of scatterer is needed. This can be done by knowing the minimum resolution and randomness of ink particles of the employed printing system (the inkjet printer, the metallic ink, and the PET film). Therefore, to estimate the minimum resolution, we printed two input digital masks based on solid circular shapes with diameters of $20 \mu \mathrm{m}$ and $30 \mu \mathrm{m}$ on PET printable film, successively. Then, the dimensions of inkjet-printed output are analyzed by using a digital microscope. This process is illustrated in Fig. 3, where the randomness of ink droplets in the inkjet-printed outputs can be observed. According to [24], the inkjet-printed output is usually constituted of two types of ink droplets: main droplets and satellite droplets (which are smaller and separated ink droplets). In our case, if we ignore the satellite droplets, the calculated resolution range of the employed printing system is from $55 \mu \mathrm{m}$ to $116 \mu \mathrm{m}$ as a first approximation.

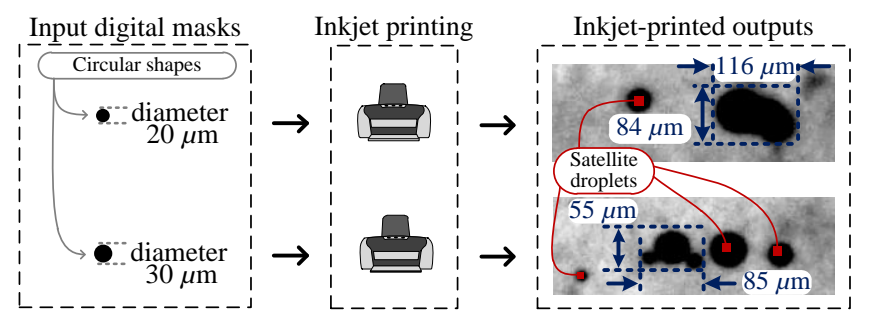

Fig. 3. Illustration of the estimation of minimum resolution and randomness in inkjet printing using Epson C88+ printer with JS-B25P silver conductive ink on a PET printable film.

Keeping the estimated resolution range in view, the input digital mask of scatterer [Fig. 2(b)] is further optimized, where we introduced a vertex-to-vertex gap $d_{s}$ of $70.7 \mu \mathrm{m}$ among the square elements as shown in Fig. 4(a). This value of $d_{s}$ is chosen in the middle of inkjet printer resolution range (i.e., from $55 \mu \mathrm{m}$ to $116 \mu \mathrm{m}$ ) to exploit the randomness inherent to inkjet printing efficiently. This further optimized input digital mask [presented in Fig. 4(a)] is then supplied to the inkjet printer, and thirty prototypes of chipless tags using one identical input digital mask are printed on PET printable film. Fig. 4(b) shows the first inkjet-printed chipless RFID tag.

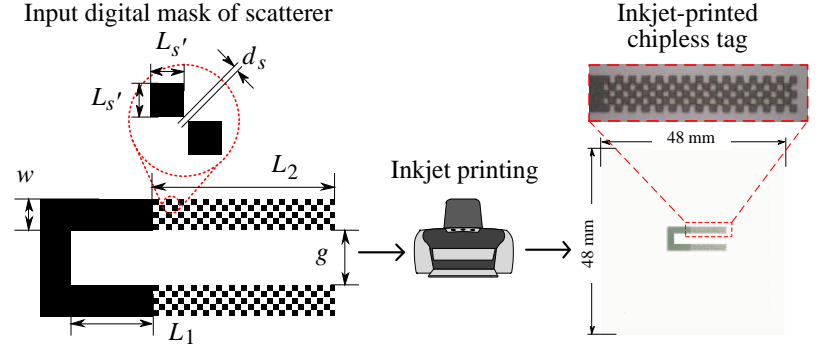

(a)

(b)

Fig. 4. Further optimization of input digital mask [from Fig. 2(b)] and inkjet printing of chipless RFID tags. (a) Further optimized input digital mask with vertex-to-vertex gap $d_{s}=70.7 \mu \mathrm{m}$ among the square elements of check pattern. The optimized length (and width) of each square geometry $L_{s^{\prime}}=0.36 \mathrm{~mm}$ and the rest of the dimensions are precisely the same as shown in Fig. 2. (b) Top view of first inkjet-printed chipless tag based on check patterned C-folded scatterer on PET printable film.

\section{AUTHENTICATION RESULTS}

For a low cost practical solution, in-house built IR-UWB chipless RFID reader [22] is used for experimental results. This IR-UWB chipless reader is developed using off-the-shelf available components. For this reason, this IR-UWB chipless RFID reader is a cost-effective apparatus with a price comparable to the conventional RFID reader employed in the supply chain management and logistics. The band of operation of this reader is $3.1 \mathrm{GHz}$ to 10.6 GHz. For the free space measurements using IR-UWB chipless reader, a co-polarization bistatic configuration inside the anechoic environment with Satimo (QH2000) quad ridged open boundary antennas $(2-32 \mathrm{GHz})$ is used as shown in Fig. 5. The chipless tags are placed at a distance $r=16.5 \mathrm{~cm}$ from the antenna. The separation distance between the two antennas is $e=2.7 \mathrm{~cm}$.

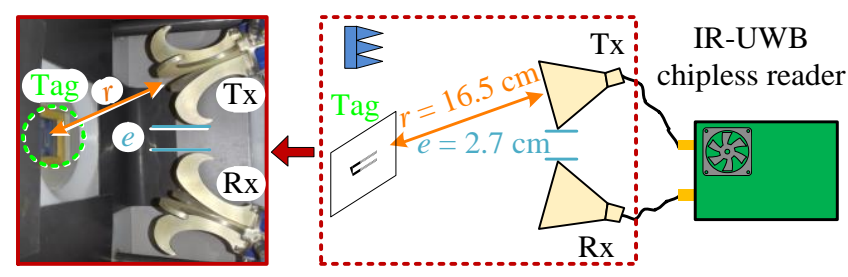

Fig. 5. Block diagram of measurement setup of bistatic configuration in an anechoic environment with IR-UWB chipless RFID reader. Inset: photograph of the measurement setup.

Each tag is measured five times successively by removing and repositioning the tag at its position during each measurement trial to check the repeatability. The measured parameter is the transmission coefficient $S_{21}$ due to the reader configuration. The post-processing (i.e., background normalization and time windowing) is applied to measured signals (for more explanation, see [12]). For instance, the time windowing for $S_{21}$ of first squares check patterned tag is shown in Fig. 6. The start time of time window (13 ns) along with window length $(7 \mathrm{~ns})$ is shown in Fig. 6. Here, the parameters of postprocessing are optimized for this specific signal (i.e., limited to $7 \mathrm{~ns}$ due to the wires reflections), as the IR-UWB reader is entirely different from VNA based reader [12], where IR-UWB uses an UWB pulse in TD. The non-windowed TD signal (i.e., measured using the measurement configuration shown in Fig. 5) exhibits 3789 data points. After time windowing, the windowed TD signal exhibits 700 data points by neglecting the padded zeros. The padded zeros do not contribute to the similarity while comparing the signals. The windowed frequency-domain (FD) signal exhibits 380 points.

The first measurements of $S_{21}$ in the form of windowed FD responses for selected tags from the population of thirty chipless 
RFID tags are shown in Fig. 7. The randomness happened during the inkjet printing process can be observed from the randomness of the position of peak apexes corresponding to the frequencies of resonances of chipless tags.

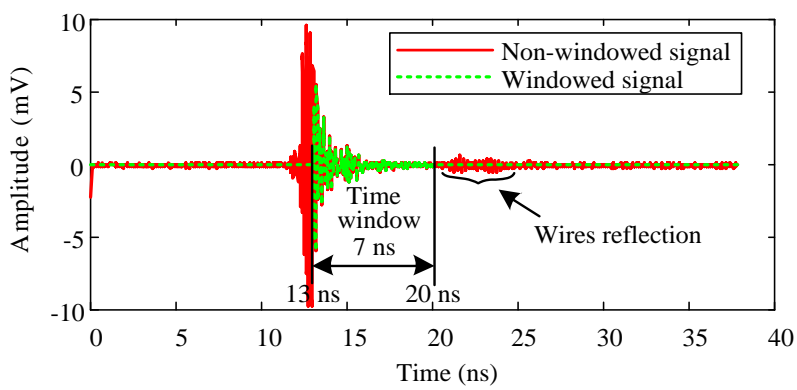

Fig. 6. Time windowing to discard the structural mode and to extract the antenna mode of $S_{21}$ of first squares check patterned tag measured with IRUWB reader. The start time of time window is $13 \mathrm{~ns}$ and the duration of time window is $7 \mathrm{~ns}$.

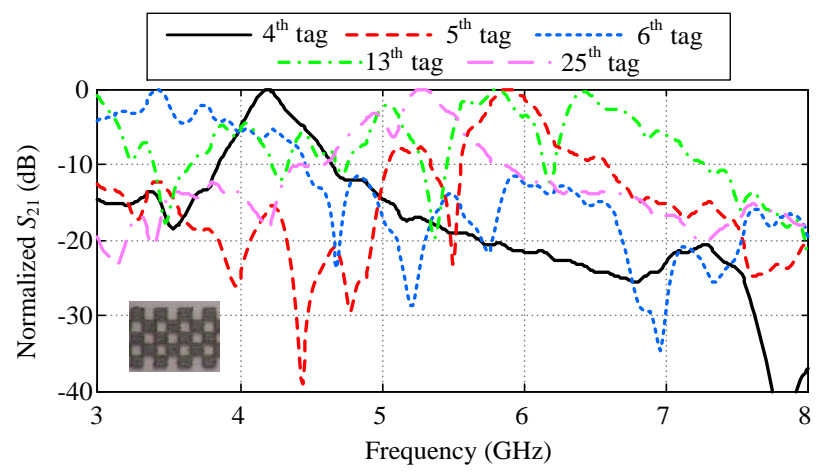

Fig. 7. First measurement of $S_{21}$ in the form of windowed FD responses for selected chipless tags from the population of thirty chipless tags. Inset corresponding zoom photograph of the inkjet-printed five squares check pattern.

On the other hand, Fig. 8 shows an example of the repeatability of measurement system by presenting the repetitive measurements of $S_{21}$ in the form of windowed FD responses for first tag from five squares check patterned $\mathrm{C}$-folded scatterer tag population using IRUWB chipless reader. It can be observed that the repetitive signals exhibit similar characteristic features (e.g., the position of peak apex), but they do not present perfect agreement. This is because of the low accuracy of IR-UWB chipless RFID reader.

After the post-treatment, all the measured signals (i.e., 30 tags $\times 5$ measurements for each tag $=150$ signals) are compared among them to calculate similarity level. For all thirty realizations of the chipless tags, the intra-tag combinations can be calculated as $30 C_{2}^{5}=300$ and the inter-tag combinations can be calculated as $C_{2}^{30 \times 5}-30 C_{2}^{5}=24750$. In PCB chipless tags based authentication [12], merely 200 points around the peak apexes (i.e., a range from $12.632 \mathrm{GHz}$ to 12.726 $\mathrm{GHz}$ ) are used for FD signals' comparison. In this proposed inkjetprinted chipless tags based authentication, full signals (with all points) are used for FD signals' comparison, as the peak apexes vary in full range from $3.4 \mathrm{GHz}$ to $6.5 \mathrm{GHz}$ (see Fig. 7). This is because the adopted square check pattern has significantly elevated the sensitivity of the scatterer to the fabrication process in this work. Fig. 9 presents the similarity analyses for inkjet-printed PET five squares check patterned tags measured using IR-UWB chipless reader. These statistical analyses are performed for both FD and TD signals as explained in [12, Fig. 3]. For FD analysis [Fig. 9(a)], intraand inter-tag probability density function (PDF) distributions are calculated using cosine similarity (CS) and for TD analysis [Fig. 9(b)], intra- and inter-tag PDF distributions are calculated using maximum valued correlation coefficient $\left(\mathrm{CC}_{\max }\right)$. The mathematical expressions of $\mathrm{CS}$ and $\mathrm{CC}_{\max }$ can be seen in [12]. Furthermore, in both FD and TD analyses, an asymmetrical double sigmoid (ADS) function [25] is used to fit the inter-tag PDF distributions, while a Gaussian (G) PDF is used to fit the intra-tag PDF distributions. It can be observed from the insets of Fig. 9(a) and (b) that the intra- and inter-tag PDF distributions slightly overlap in both FD and TD analyses. On the other hand, the central tendency, for example, the mean values of the intra-tag PDF distributions of both FD and TD analyses are close to unity, which validates the repeatability of each tag. The spreads of the intra-tag PDF distributions below the unity are due to the uncertainties (systematic error and random error) from the measurement bench and less accuracy in repeatability of IRUWB reader (see Fig. 8).

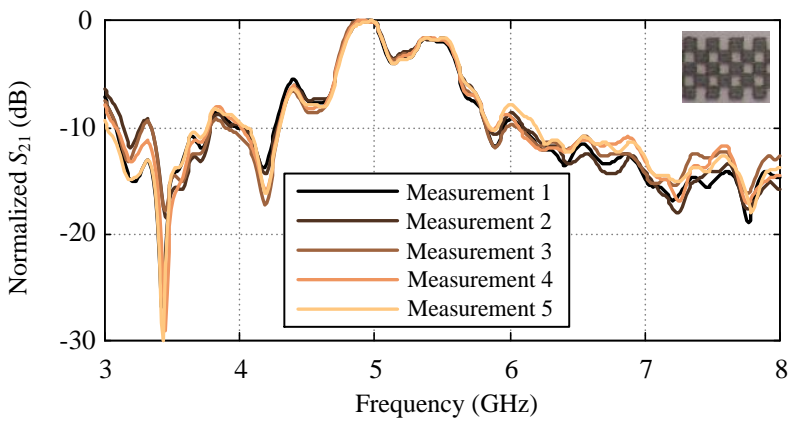

Fig. 8. Five repetitive measurements of $S_{21}$ in the form of windowed FD responses for first tag from five squares check patterned C-folded scatterer tags population using IR-UWB chipless reader. Inset: corresponding zoom photograph of the inkjet-printed pattern.

Finally, the probability of false negative (PFN) and the probability of false positive (PFP) curves are calculated for both the actual PDF distributions and the fitted PDF distributions. The error rates are calculated from the actual PDF distributions as well as from the fitted PDF distributions as shown in Fig. 9(c).

For the actual PDF distributions, the calculated probability of error (PE) for FD analysis is $\mathrm{PE}_{\mathrm{FD}}^{\text {dist }}=2.3 \%$ and the calculated $\mathrm{PE}$ for TD analysis is $\mathrm{PE}_{\mathrm{TD}}^{\text {dist }}=2.7 \%$ [see the lines without markers in Fig. 9(c)]. If we use the fitted PDF distributions, the calculated PE for FD analysis is $P E_{\mathrm{FD}}^{\mathrm{fit}}=1.1 \%$ and the calculated $\mathrm{PE}$ for $\mathrm{TD}$ analysis is $\mathrm{PE}_{\mathrm{TD}}^{\text {fit }}=3 \%$ [see the lines with markers in Fig. 9(c)]. It can be observed that $\mathrm{PE}_{\mathrm{TD}}^{\mathrm{fit}}>\mathrm{PE}_{\mathrm{TD}}^{\text {dist }}$, that is due to the overestimation of distribution from ADS fitting function applied to the inter-tag PDF distributions. This underestimation or overestimation of inter-tag PDF distributions near tails (between 0.8 and 1) emerges due to the uni PDF fitting method. In this paper, we present a simple method to model the authentication performance of the system. However, for the precise modeling of the system, the more complex fitting method such as using multi PDFs can be used. On the other hand, we have also provided the results using the actual PDF distributions.

The average PEs for the fingerprint verification system ranging from $2.07 \%$ to $4.03 \%$ found in [26] are comparable to the results obtained by using IR-UWB chipless reader (e.g., PE $E_{\mathrm{FD}}^{\text {dist }}=2.3 \%$ and $\mathrm{PE}_{\mathrm{TD}}^{\text {dist }}=2.7 \%$ ).

For an accurate performance characterization and the comparison with IR-UWB reader, experimental measurements using a VNA (Agilent 5222A) are also performed. These measurements are in the same co-polarization bistatic configuration inside the anechoic environment with the same the antennas, $r$ and $e$ as shown in Fig. 5. 


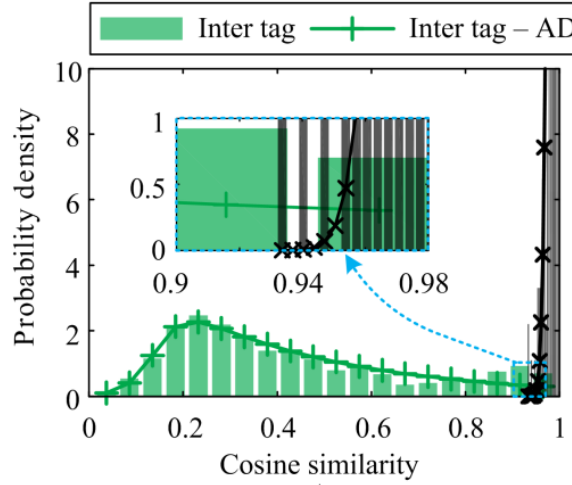

(a)

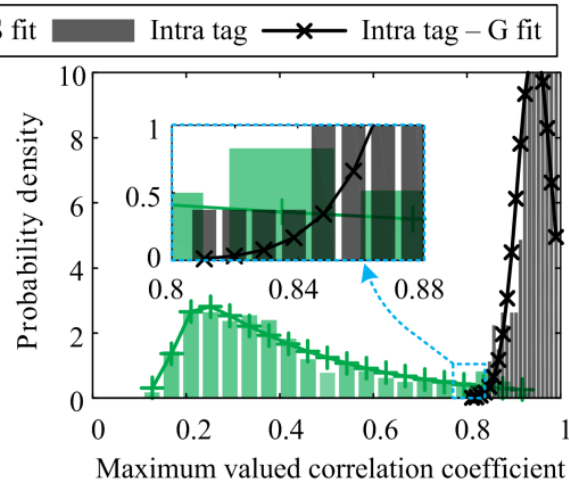

(b)

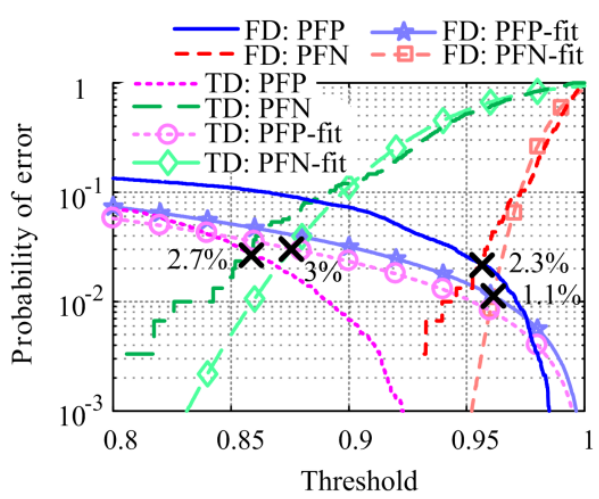

(c)

Fig. 9. Similarity analysis for the inkjet-printed PET five squares patterned tags with $d_{s}=70.7 \mu$ m measured using IR-UWB chipless reader. (a) Intra- and inter-tag cosine similarity PDF distributions for FD analysis. (b) Intra- and inter-tag maximum valued correlation coefficient PDF distributions for TD analysis. (c) Probabilities of false positive and false negative for both FD and TD analyses.

The source power of VNA is equal to $-5 \mathrm{dBm}$. The frequency sweep ranging from 3 to $8 \mathrm{GHz}$ with 10001 points is used. The experimental measurement procedure and the measurement counts are also the same as discussed above for IR-UWB reader. In this VNA based reader, the measured quantity is the reflection coefficient $S_{11}$, which is to be more promising for a practical situation due to the use of only one antenna (fewer resources). The post-processing (i.e., background normalization and time windowing) is also applied, where the early part of the TD signals up to $2.2 \mathrm{~ns}$ is discarded and the subsequent part of $10 \mathrm{~ns}$ is extracted. The effective length of the windowed TD signal is of 100 points by neglecting the padded zeros. The windowed FD signal exhibits 9800 points. For the actual PDF distributions in both FD and TD analyses, the probabilities of error $\left(\mathrm{PE}_{\mathrm{FD}}^{\mathrm{dist}}\right.$ and $\left.\mathrm{PE}_{\mathrm{TD}}^{\mathrm{dist}}\right)$ are in the range of $10^{-3}$ to $10^{-2}$ (i.e., $0.01 \%$ to $0.1 \%)$. From the fitted PDF distributions, $P E_{\mathrm{FD}}^{\mathrm{fit}}=0.013 \%$ and $\mathrm{PE}_{\mathrm{TD}}^{\mathrm{fit}}=0.003 \%$. Such levels of $\mathrm{PE}$ are quite insignificant as compared to the best average PE of $0.05 \%$, among different fingerprint evaluation campaigns found in [27].

The difference between the performance of IR-UWB chipless reader and VNA based chipless reader is that TD signal from low cost IR-UWB chipless reader is less accurate than FD signal from highly costly and highly accurate VNA (i.e., known to have higher resolution). The performance of IR-UWB chipless reader is ten times lower than VNA based reader, but the cost of IR-UWB chipless reader a hundred times less than VNA based chipless reader.

To show the effect of the number of square geometries and the vertex-to-vertex gap $d_{s}$ on the authentication performance, we printed populations of thirty tags based on three squares check pattern with three values of $d_{s}: 21.2 \mu \mathrm{m}, 42.4 \mu \mathrm{m}$, and $70.7 \mu \mathrm{m}$. Table I shows an evolution of PE with $d_{s}$ for the three squares patterned C-folded design. Here, too, for accurate characterization, free space measurements are performed VNA based chipless reader with exact same equipment and parameters (e.g., VNA, the antennas, the cables, the distances, the output power, the frequency sweeping, the number of points, and anechoic environment) as discussed above. $S_{11}$ of each tag is measured five times with removing and repositioning manner. The rest of the post-treatment on the measured signals (background normalization and time windowing along with chosen parameters for time window) is the same as discussed above for VNA based chipless reader in this section. Similarity, PDF distributions corresponding to FD and TD are calculated using CS and $\mathrm{CC}_{\max }$, respectively. Finally, the PEs are calculated using actual $\mathrm{PDF}$ distributions $\mathrm{PE}_{\mathrm{FD}}^{\text {dist }}$ and $\mathrm{PE}_{\mathrm{TD}}^{\text {dist }}$ (not the fitted PDF distributions). From the results in Table I, an increasing trend of the performance as $d_{s}$ increases can be observed. At $d_{s}=70.7 \mu \mathrm{m}$, the optimum exploitation of randomness inherent to inkjet printing can be observed with $\mathrm{PE}_{\mathrm{FD}}^{\text {dist }}=0.41 \%$ and $\mathrm{PE}_{\mathrm{TD}}^{\text {dist }}=0.33 \%$ (see the highlighted values in Table I). On the other hand, with $d_{s}=70.7 \mu \mathrm{m}$, the five squares pattern outperforms the three squares pattern, as the obtained PEs from five squares check patterned tags $\left(P E_{\mathrm{FD}}^{\text {dist }}\right.$ and $\mathrm{PE}_{\mathrm{TD}}^{\text {dist }}$ are ranging from $0.01 \%$ to $0.1 \%$ ) are smaller in magnitude than the obtained PEs from three squares check patterned tags $\left(\mathrm{PE}_{\mathrm{FD}}^{\text {dist }}=0.41 \%\right.$ and $\left.\mathrm{PE}_{\mathrm{TD}}^{\text {dist }}=0.33 \%\right)$. This is due to larger surface area is exposed to the inkjet printing in five squares pattern that in turn give rise to larger variations of proximate couplings in the design.

TABLE I

Evolution of THE Probability OF ERRor With Changes IN VERTEX-TOVertex GaP $d_{s}$ AMONG the SQuares For the Three SQuares PatTerned C-FOLDED DESIGN.

\begin{tabular}{ccc}
\hline \hline \multirow{2}{*}{$d_{s}(\mu \mathrm{m})$} & \multicolumn{2}{c}{ Probability of error $(\%)$} \\
& FD & TD \\
\hline 21.2 & 6 & 5.46 \\
42.4 & 3 & 2.24 \\
70.7 & 0.41 & 0.33 \\
\hline \hline
\end{tabular}

\section{FLOW OF IMPLEMENTATION OF PROPOSED ChIPLESS AuthentiCATION Method}

In this Section, the procedure for the employment of chipless RFID tags for authentication is explained. First, a single input digital mask is supplied to the inkjet printer to realize a population of chipless RFID tags. Soon after the printing of chipless tags, RF fingerprint of each tag is measured individually using a measurement setup based on a controlled anechoic environment and a chipless RFID reader as schematized in [12, Fig. 2]. Then, all measurements are stored to construct a database of authenticity. The possible applications of the proposed method are, for example, the chipless tags can directly be printed on a document or hidden in the product packaging. Then, these products or documents are sold to the consumer. The printing of chipless tag on a document is like providing a simple, low cost and secure solution of keeping the tracks of paper documents, books, and packages for the print industry as discussed in [28]. When a consumer requires the authentication of the document or the product, a second measurement needs to be done to evaluate the possible match in the database of authenticity. In the case of the security document, the second measurement (i.e., for the comparison with database) can be 
taken without any destructive operation. Conversely, if the chipless tag is hidden in the product packaging, an authorized destructive extraction of chipless tag is required. The chipless tag must only be removed by an authorized person from the authentication solution provider, where the solution provider first investigate that the chipless tag has never been removed earlier. The idea of utilizing the chipless tags for authentication of manufactured products might not be conceived as RFID labels that can be attached, removed and then reattached to a product by the consumer. The chipless authentication can be considered as numerous high-level tools (e.g., high-level security seal) that need to be permanently affixed to the product. Any forgery to chipless tag would change the backscattered response resulting in the product as a counterfeit. After removal of chipless tag for authentication, a new tag can be added for future authentication. The details about the constructions of a database of authenticity and the procedure for authentication are explained in [12]. It is important to note that the practical implementation of the proposed authentication method, a controlled anechoic measurement environment is needed which can be realized in a small box. One can say that such a controlled environment is a limitation of the proposed authentication method. However, a controlled environment might be beneficial to ensure the high security level of the proposed authentication method, because its details can be kept secret. The obtained results presented in Section $\mathrm{V}$ are from the signals measured in the room size anechoic environment. However, the small anechoic environment would provide comparable results because the purpose of both environments is the same which is to provide isolation from the outside environment.

The proposed authentication method has a significant advantage of the possibility to hide the chipless RFID tag entirely by virtue of RF.

\section{CONCLUSION}

The opportunity to exploit the process randomness inherent to inkjet printing for the generation of unique EM signals was discussed in this paper. These unique signals were then employed to provide a highly secure authentication solution. The design of conventional Cfolded scatterer was optimized by introducing discrete square check patterns along the arms of C-folded scatterer to elevate its sensitivity to inkjet printing. Then, the proposal was validated by showing the performance of the optimized sensitive check patterned C-folded chipless tags realized on the PET printable film measured using a low-cost IR-UWB chipless reader as well as a highly accurate VNA based chipless reader. The achieved PEs are comparable to the various fingerprint evaluation campaigns found in the literature. The advantage of the proposed chipless authentication is that contrary to classical image based authentication approach (like a fingerprint), the chipless tag can entirely be hidden (potentially without performance degradation) for confidentiality and the product integrity of the high-end luxury products.

\section{REFERENCES}

[1] G. Power, "Anti-counterfeit Technologies for the Protection of Medicines," World Health Organization, Geneva, Switzerland, 2008.

[2] F. Bourrieres and C. Kaiser, "Method and device for reading authentication means and adapted identification means," US7380128B2, 27-May-2008.

[3] M. Schmitt-Lewen, B. Vosseler, K.-H. Walther, and S. Akkaya, "Method for producing a security feature on a flat substrate," US20090274298A1, 05-Nov-2009.

[4] M. Greene and G. W. Hurley, "Method for forming a radio frequency responsive target and apparatus for verifying the authenticity of same," US6471878 B1, 29-Oct-2002.
[5] E. Perret, A. Vena, S. Tedjini, Y. Boutant, and C. Halope, "Item comprising a barcode with an electromagnetic signature," US9697446 (B2), EP2864935 (A2), 2012.

[6] K. Yang, D. Forte, and M. M. Tehranipoor, "UCR: An unclonable chipless RFID tag," in Proc. IEEE Int. Symp. Hardw. Oriented Secur. Trust (HOST), Washington, DC, USA, 2016, pp. 7-12.

[7] C. Herrojo, J. Mata-Contreras, A. Núñez, F. Paredes, E. Ramon, and F. Martín, "Near-Field Chipless-RFID System With High Data Capacity for Security and Authentication Applications," IEEE Trans. Microw. Theory Techn., vol. 65, no. 12, pp. 5298-5308, Dec. 2017.

[8] F. J. J. Romero, J. V. GARCÍA, L. J. F. FERNÁNDEZ, A. J. Gamo, and B. M. Á. RODRÍGUEZ, "Use of radiofrequency wave absorbing markers for the authentication of security documents," EP2694600B1, 01-Nov-2017.

[9] Z. Ali et al., "Potential of chipless authentication based on randomness inherent in fabrication process for RF and THz," in Proc. 11th Eur. Conf. Antennas Propag. (EUCAP), Paris, France, 2017, pp. 2559-2563.

[10] Z. Ali et al., "Chipless RFID Tag Discrimination and the Performance of Resemblance Metrics to be used for it," in Proc. IEEE MTT-S Int. Microw. Symp. (IMS), Philadelphia, PA, USA, 2018, pp. 363-366.

[11] Z. Ali et al., "Detection of Minimum Geometrical Variation by FreeSpace-Based Chipless Approach and its Application to Authentication," IEEE Microw. Wireless Compon. Lett., vol. 28, no. 4, pp. 323-325, 2018.

[12] Z. Ali et al., "Detection of Natural Randomness by Chipless RFID Approach and its Application to Authentication," IEEE Trans. Microw. Theory Techn., vol. 67, no. 9, pp. 3867-3881, 2019.

[13] G. DeJean et al., "RFDNA: A wireless authentication system on flexible substrates," in Proc. IEEE 61st Electron. Compon. and Technol. Conf. (ECTC), Lake Buena Vista, FL, USA, 2011, pp. 1332-1337.

[14] Y. Boutant, J. M. Becker, and T. Fournel, "Method for Extracting Random Signatures from a Material Element and Method for Generating a Decomposition Base to Implement the Extraction Method," CA2953689 (A1), 28-Jun-2007.

[15] I. M. Hutchings and G. D. Martin, Eds., Inkjet Technology for Digital Fabrication. John Wiley \& Sons, 2012.

[16] P. C. Duineveld, "The stability of ink-jet printed lines of liquid with zero receding contact angle on a homogeneous substrate," Journal of Fluid Mechanics, vol. 477, pp. 175-200, 2003.

[17] D. Redinger, S. Molesa, S. Yin, R. Farschi, and V. Subramanian, "An ink-jet-deposited passive component process for RFID," IEEE Trans. Electron. Devices, vol. 51, no. 12, pp. 1978-1983, Dec. 2004.

[18] J. F. Salmerón et al., "Properties and Printability of Inkjet and ScreenPrinted Silver Patterns for RFID Antennas," Journal of Electronic Materials, vol. 43, no. 2, pp. 604-617, Feb. 2014.

[19] R. Garg, I. Bahl, and M. Bozzi, Microstrip Lines and Slotlines, Third Edition: Artech House, 2013.

[20] O. Rance, R. Siragusa, P. Lemaître-Auger, and E. Perret, "Contactless Characterization of Coplanar Stripline Discontinuities by RCS Measurement," IEEE Trans. Antennas Propag., vol. 65, no. 1, pp. 251257, Jan. 2017.

[21] A. Vena, E. Perret, and S. Tedjini, "Chipless RFID Tag Using Hybrid Coding Technique," IEEE Trans. Microw. Theory Techn., vol. 59, no. 12, pp. 3356-3364, Dec. 2011.

[22] M. Garbati, E. Perret, and R. Siragusa, Chipless RFID Reader Design for Ultra-Wideband Technology: Design, Realization and Characterization. Elsevier Science, 2018.

[23] O. Rance, R. Siragusa, P. Lemaitre-Auger, and E. Perret, "Toward RCS Magnitude Level Coding for Chipless RFID," IEEE Trans. Microw. Theory Techn., vol. 64, no. 7, pp. 2315-2325, Jul. 2016.

[24] S. D. Hoath, S. Jung, W. K. Hsiao, and I. M. Hutchings, "How PEDOT:PSS solutions produce satellite-free inkjets," Organic Electronics, vol. 13, no. 12, pp. 3259-3262, Dec. 2012.

[25] J. Hjelt, H. Collan, M. Yakushev, and K. Hjelt, "The role of spatial potential fluctuations in the shape of the PL bands of multinary semiconductor compounds," Physica Scripta, vol. 1999, no. T79, p. 179, 1999.

[26] R. Cappelli, D. Maio, D. Maltoni, J. L. Wayman, and A. K. Jain, "Performance evaluation of fingerprint verification systems," IEEE Trans. Pattern Anal. Mach. Intell., vol. 28, no. 1, pp. 3-18, Jan. 2006.

[27] D. Maltoni, D. Maio, A. Jain, and S. Prabhakar, Handbook of Fingerprint Recognition, 2nd ed. London: Springer-Verlag, 2009.

[28] D. F. Christofferson, E. J. Anderson, J. C. Geres, and W. T. Graushar, "Resonator use in the print field," US7506813B2, 24-Mar-2009. 